\title{
A Comparative study to evaluate the efficacy of Supervised Exercise Program and Cyriax Physiotherapy on pain and function in Lateral Epicondylitis
}

\section{Gouri Kalaskar}

Ravi Nair Physiotherapy College, Datta Meghe Institute of Medical Sciences, Sawangi (meghe), Wardha, Maharashtra, India https://orcid.org/0000-0002-6270-5538

\section{R. K. Sinha}

Mahalaxmi College Of Physiotherapy and Rehabilitation Center Raigaon, Satara, Maharashtra, India Pratik Phansopkar ( $\nabla$ drpratik77@gmail.com )

Ravi Nair Physiotherapy College, Datta Meghe Institute of Medical Sciences, Sawangi (Meghe), Wardha, Maharashtra, India https://orcid.org/0000-0003-3635-8840

\section{Method Article}

Keywords: Cyriax Physiotherapy, Lateral Epicondylitis, Supervised Exercise Programme, VAS, TEFS

Posted Date: July 12th, 2021

DOI: https://doi.org/10.21203/rs.3.pex-1484/v1

License: (9) This work is licensed under a Creative Commons Attribution 4.0 International License. Read Full License

Version of Record: A version of this preprint was published at Journal of Pharmaceutical Research International on November 18th, 2021. See the published version at https://doi.org/10.9734/jpri/2021/v33i50B33431. 


\section{Abstract}

Background: A typical musculotendinous degenerative condition of the extensors cause the humerus lateral epicondyle is known as Lateral epicondylitis. Various treatment methods are used in treating lateral epicondylitis.

Methods: Thirty Participants with Lateral Epicondylitis shall be recruited in a comparative experimental study. Subjects will be randomized to either (1) Supervised Exercise Programme group, or (2) Cyriax Physiotherapy group. Over a 4-week time period, a 3 times in week for the total of 12 sessions, immediately following baseline assessment and randomization, subjects in both groups will receive Extensor Carpi Radialis Brevis muscle's static stretching along with the wrist extensor's eccentric strengthening and Ultrasound and transverse deep friction massage for 10 min with Mill's manipulation and Ultrasound respectively.

Discussion: Effectiveness of the interventions on the pain and the functional improvement will be assessed by visual analogue scale and the Tennis Elbow Function Scale respectively.

\section{Introduction}

The disorder essentially inside the radio humeral joint with constant impairing pain in the elbow, is named as tendonitis, tennis elbow, lateral epicondyalgia or lateral epicondylitis ${ }^{1}$. The precise explanation for tendonitis isn't however identified. It is quite common in people whose jobs demand recurring forearm movements (e.g., court game sportsmen and woodworkers) ${ }^{2}$. It's normally because of additional fast, wearisome, recurrent eccentric contractions and gliding joint griping activities. It normally affects dominant arm. The typical amount of associate degree episode of epicondylitis in span of six months and two years. In tennis elbow macroscopic and microscopic lesions appears within the Extensor Carpi Radialis Brevis (ECRB) muscle ${ }^{3}$. Histologic analysis of excised pathologic ECRB tendons does not show acute inflammatory cells, implying that lateral epicondylitis is not a primary inflammatory disease. Instead, immature fibroblasts and disorganised, nonfunctional vascular elements have invaded the tissue. Angiofibroblastic hyperplasia was invented by Nirschl and Pettrone to characterise this granulation-like tissue $e^{4}$. The radial recurrent artery was established by Schneberger and Masquelet as the dominant vascular supply to the ECRB tendon ${ }^{5}$. Two hypovascular zones were discovered after further investigation: one at the lateral epicondyle and the other $2-3 \mathrm{~cm}$ distal to the extensor insertion. $4 \mathrm{~A}$ common cause of lateral epicondylitis is repetitive microtrauma from overuse of the wrist extensors. A promising but unproven cause of lateral epicondylitis is microtrauma in the context of hypovascularity, which prevents healing ${ }^{6}$.

The primary complaints in lateral epicondylitis includes lessened strength in grip, inflated pain and reduced functional activity. The diagnosis established by the examination which might evoke the pain, over the lateral epicondyle facet, tenderness on touch, finger extension with resistance, wrist joint extension with resistance, and wrist joint flexion passive in nature (cozen's test) ${ }^{7}$. Although various 
studies are directed on this clinical condition treatment, until now the foremost effectual management plan isn't in agreement whether or not it's standard or surgical. Since lateral epicondylitis is a clinical condition, radiological testing is rarely necessary and reserved for those who do not improve. X-ray is the first line of inquiry when there is a suggestion of radio-capitellar osteoarthritis or other bony pathology ${ }^{8}$.

To evaluate the efficacy of the Supervised Exercise Programme and therefore the Cyriax Physiotherapy on pain and functional improvement in tennis elbow.

This study will be carried out in the Musculoskeletal Physiotherapy OPD of Ravi Nair Physiotherapy College, Sawangi (Meghe), Wardha, after approval from Institutional Ethics Committee of Datta Meghe Institute Of Medical Sciences, Deemed to be University. Before inclusion, all the participants will be informed regarding the aim and procedure of research. Those participants who will meet the inclusion criteria must give the written informed consent. In a comparative study, the participants ( $N=30)$ diagnosed with lateral epicondylitis (LE) will be enrolled for 4 weeks ${ }^{9}$.

A comparative study in which the patients will be randomized into two group independent design. Group I will be Supervised Exercise Programme group and Group II will be assigned with Cyriax physiotherapy which basically includes Deep Fraction Massage and Mill's manipulation.

Based on the criteria of inclusion and exclusion participants will be involved in the study. The subjects in the 20-50 years of age group of both the genders will be included. Subjects who are going to be having pain on passive flexion of wrist with the elbow extension and wrist pain on resisted extension incorporated in study ${ }^{10}$. Also the participants with chronic lateral epicondylitis and will be having tenderness on the lateral epicondyle of humerus on palpation will be encompassed.

Subjects who will be having cervical radiculopathy, neurological impairments, any preceding trauma to elbow region, any surgeries to elbow region and elbow pain acquired from any other pathology than tennis elbow (lateral epicondylitis) will be eliminated from the study. The subjects who are getting to be having neuromuscular diseases, peripheral nervous disorder and any of them who are getting to be receiving corticosteroid injection in previous 6 months before the study period begins, will not be considered in to avoid further complications.

Each patient will be required to complete 4 weeks of interventions like supervised exercise programme and cyriax physiotherapy after enrolment in the study.

The patients who are already undergoing rehabilitation in our IPD or comimg to the physiotherapy OPD and diagnosed with lateral epicondylitis will be systematically assessed for the eligibility in the study according to the inclusion and exclusion criteria. After fulfilling the eligibility criteria for their enrollment in the study, the informed patient consent will be cumulated taken after elaborating the purpose and procedure of the study. 
This comparative study is an experimental two-group design that examines efficacy of the two interventions - the supervised exercise programme and the physiotherapy of cyriax in lateral epicondylitis (tennis elbow) treatment ${ }^{11}$. 30 participants will be enrolled to the group I and group II and will be randomized by cheat method.

All the participants of administered supervised exercise programme will undergo static stretching of Extensor Carpi Radialis Brevis (ECRB) in a flash followed by the wrist extensors eccentric strengthening and the position of the steady stretch will be held for 30-45 seconds, this intervention will be carried out 3 counts prior and 3 counts after the segment of strengthening eccentric in nature treatment for 6 repetitions in total ${ }^{12}$. In each repetition of stretching There will be 30 seconds of interim.

All the participants will get Cyriax physiotherapy that involves transverse deep friction massage for ten minute which will be straight away accompanied by Mill's manipulation/ manoeuvre. With the side of the fingertip of the thumb on the area of tenderness after palpating the anterior and lateral side of the lateral epicondylar surface of the humerus transverse deep friction massage will be applied. After achieving the numbing effect, the tendon is prepared for Mill's manipulation. Mill's manoeuvre is thrust of a low in

amplitude and high in velocity in the end range of elbow extension. It will be single application ${ }^{13}$.

1) Visual Analogue Scale - VAS will evaluate the pain, which is a horizontal bar of $10 \mathrm{~cm}$ with two ends zero $\mathrm{cm}$ is a "minimum imaginable pain" and ten $\mathrm{cm}$ "worst pain imaginable". Patient will have to draw out a upright line on the horizontal scale according to their present level of pain.

2) Tennis Elbow Function Scale (TEFS) - The Functional activity of elbow will be evaluated on Tennis Elbow function Scale which have ten group of activities and their rating will be based on discomfort i.e. 'No discomfort' to 'Extreme discomfort'. This scale is scores in elation with VAS in combination for pain related function.

The assessment data will be collected from a predetermined spreadsheet with the baseline characteristics variable. The hard copies of assessment forms and signed consent forms will be stored securely at the study site. Data collection and documentation will be done under the guidance of the principal investigators. The study documentation will be evaluated thoroughly for accuracy.

\section{Reagents}

\section{Equipment}

\section{Procedure}

1. Recruitment of 30 subjects after screening by inclusion and exclusion criteria.

2. Obtain informed consent and medical history from subjects. 
3. Perform baseline assessment.

4. Randomize subjects in two groups namely Group I and Group II containing 15 subjects each.

5. Perform supervised exercise program for 4 weeks in Group I subjects which shall include static stretching of ECRB for 30-45 seconds followed by eccentric strengthening of wrist extensors - 3 repetitions 3 times a week.

6. Perform Cyriax physiotherapy for 4 weeks in Group II subjects which shall include deep transverse friction massage for 10 minutes and Mill's manipulation 3 times a week.

7. Perform assessment after 4 weeks of treatment.

8. Analyze statistically.

\section{Troubleshooting}

\section{Time Taken}

\section{Anticipated Results}

\section{References}

1. Viswas, R., Ramachandran, R. \& Korde Anantkumar, P. Comparison of Effectiveness of Supervised Exercise Program and Cyriax Physiotherapy in Patients with Tennis Elbow (Lateral Epicondylitis): A Randomized Clinical Trial. Sci. World J. 2012, 1-8 (2012).

2. Cho, Y.-T., Hsu, W.-Y., Lin, L.-F. \& Lin, Y.-N. Kinesio taping reduces elbow pain during resisted wrist extension in patients with chronic lateral epicondylitis: a randomized, double-blinded, cross-over study. BMC Musculoskelet. Disord. 19, 193 (2018).

3. Blanchette, M.-A. \& Normand, M. C. Impairment assessment of lateral epicondylitis through electromyography and dynamometry. 11 .

4. Nirschl, R. P. \& Sobel, J. Conservative Treatment of Tennis Elbow. Phys. Sportsmed. 9, 43-54 (1981).

5. Schneeberger, A. G. \& Masquelet, A. C. Arterial Vascularization of the Proximal Extensor Carpi Radialis Brevis Tendon: Clin. Orthop. 398, 239-244 (2002).

6. Bagayoko, N. D. \& Brockmeier, S. F. Current controversies in the management of lateral epicondylitis. Curr. Orthop. Pract. 23, 480-485 (2012). 
7. Shamsoddini, A. \& Hollisaz, M. T. Effects of Taping on Pain, Grip Strength and Wrist Extension Force in Patients with Tennis Elbow. Trauma Mon. 18, 71-74 (2013).

8. Speers, C. J., Bhogal, G. S. \& Collins, R. Lateral elbow tendinosis: a review of diagnosis and management in general practice. Br. J. Gen. Pract. 68, 548-549 (2018).

9. Nagrale, A. V., Herd, C. R., Ganvir, S. \& Ramteke, G. Cyriax Physiotherapy Versus Phonophoresis with Supervised Exercise in Subjects with Lateral Epicondylalgia: A Randomized Clinical Trial. J. Man. Manip. Ther. 17, 171-178 (2009).

10. Knutsen, E. J. et al. Factors Associated With Failure of Nonoperative Treatment in Lateral Epicondylitis. Am. J. Sports Med. 43, 2133-2137 (2015).

11. Dimitrios, S. Lateral elbow tendinopathy: Evidence of physiotherapy management. World J. Orthop. 7, 463 (2016).

12. Stasinopoulos, D. \& Johnson, M. I. It may be time to modify the Cyriax treatment of lateral epicondylitis. J. Bodyw. Mov. Ther. 11, 64-67 (2007).

13. Vijay Kage, A. J. P. Effectiveness of Cyriax Physiotherapy in Subjects with Tennis Elbow. J. Nov. Physiother. 3, (2013).

14. Stasinopoulos, D. \& Stasinopoulos, I. Comparison of effects of Cyriax physiotherapy, a supervised exercise programme and polarized polychromatic non-coherent light (Bioptron light) for the treatment of lateral epicondylitis. Clin. Rehabil. 20, 12-23 (2006). 